\title{
MS31-P08 | CONFORMATIONAL SWITCHING IN SINGLE CRYSTALS OF A CALIX[4]RESORCINARENE
}

Mirzaei, Saber (Marquette University, Milwaukee, USA); Timerghazin, Qadir (Marquette University, Milwaukee, USA); Lindeman, Sergey (Marquette University, Milwaukee, USA)

Freshly crystallized 0,O-dipropyl-4-methyl-calix[4]resorcinarene has a boat conformation with -OPr groups oriented perpendicular to the benzene rings because of sterical hindrances. At slow cooling, a single-crystal-tosingle-crystal phase transition takes place around $240 \mathrm{~K}$ with inversion of orientation of some -OPr groups and mutual shift of the molecules in the crystal. Further cooling to $100 \mathrm{~K}$ results in complete ordering of the originally heavily disordered alkyl chains. Also, after few days in the mother liquer, the crystals of the original boat conformer redissolve and form crystals of a chair conformer. 